\title{
Helioseismic response to X2.2 solar flare of February 15, 2011
}

\author{
A.G. Kosovichev \\ W. W. Hansen Experimental Physics Laboratory, Stanford University, Stanford, CA 94305, USA
}

\begin{abstract}
The X2.2-class solar flare of February 15, 2011, produced a powerful 'sunquake' event, representing a helioseismic response to the flare impact in the solar photosphere, which was observed with the HMI instrument on the Solar Dynamics Observatory (SDO). The impulsively excited acoustic waves formed a compact wavepacket traveling through the solar interior and appearing on the surface as expanding wave ripples. The initial flare impacts were observed in the form of compact and rapid variations of the Doppler velocity, line-of-sight magnetic field and continuum intensity. These variations formed a typical two-ribbon flare structure, and are believed to be associated with thermal and hydrodynamic effects of high-energy particles heating the lower atmosphere. The analysis of the SDO/HMI and X-ray data from the Ramaty High Energy Solar Spectroscopic Imager (RHESSI) shows that the helioseismic waves were initiated by the photospheric impact in the early impulsive phase, observed prior to the hard $\mathrm{X}$-ray $(50-100 \mathrm{keV})$ impulse, and were probably associated with atmospheric heating by relatively low-energy electrons $(\sim 6-50 \mathrm{keV})$ and heat flux transport. The impact caused a short motion in the sunspot penumbra prior to the appearance of the helioseismic wave. It is found that the helioseismic wave front traveling through a sunspot had a lower amplitude and was significantly delayed relative to the front traveling outside the spot. These observations open new perspectives for studying the flare photospheric impacts and for using the flare-excited waves for sunspot seismology.
\end{abstract}

Subject headings: Sun: activity - Sun: flares - Sun: helioseismology - Sun: oscillations - sunspots

\section{Introduction}

'Sunquakes', the helioseismic response to solar flares, are caused by strong localized hydrodynamic impacts in the photosphere during the flare impulsive phase. The helioseismic waves are observed directly as expanding circular-shaped ripples on the solar surface, which can be detected in Dopplergram movies and as characteristic ridges in time-distance diagrams, (Kosovichev \& Zharkova, 1998; Kosovichev, 2006a, b) or indirectly by calculating the distribution of an integrated acoustic emission (Donea et al., 1999; Donea \& Lindsey, 2005). 
Solar flares are sources of high-temperature plasma, strong hydrodynamic motions and heat flux in the solar atmosphere. Perhaps, in all flares such perturbations generate acoustic waves traveling through the interior. However, only in some flares is the impact sufficiently localized and strong to produce the seismic waves with the amplitude above the convection noise level. The sunquake events with expanding ripples are relatively rare, and have been observed only in some high-M and X-class flares. The last previous observation of the seismic waves was reported for X1.2 flare of January 15, 2005.

It has been found in the initial July 9, 1996, flare observations (Kosovichev \& Zharkova, 1998) that the hydrodynamic impact in the photosphere ('sunquake source') followed the hard X-ray flux impulse, and hence, the impact of high-energy electrons. They suggested that the mechanism of sunquakes can be explained by a hydrodynamic 'thick-target' model of solar flares (Kostiuk \& Pikelner, 1975). Several other mechanisms of the helioseismic response, including impact by high-energy protons and back-warming radiation heating (e.g. Donea \& Lindsev, 2005; Zharkova \& Zharkov, 2007), and also due to magnetic field variations (Hudson et al., 2008). However, the mechanism, which converts a part of the flare energy and momentum into the helioseismic acoustic waves, is currently unknown. It is also unknown why only some flares generate largeamplitude waves observed as ripples or enhanced acoustic emission (see a review of Hudson, 2011, for a recent discussion and references).

Most of the previous observations of sunquakes were obtained with the Michelson Doppler Imager instrument on SOHO. However, the full-disk observations with the full $2 \mathrm{arcsec} / \mathrm{pixel} \mathrm{reso-}$ lution suitable for flare studies were obtained uninterruptedly only for 2 months a year. Thus, many flares were not observed, and the statistics of sunquakes and their relation to the flare properties were not established. Except short eclipse periods in March and September, the Solar Dynamics Observatory launched in February 2010 provides uninterrupted observations of the Sun. The Helioseismic and Magnetic Imager (HMI) on SDO observes variations of intensity, magnetic field and plasma velocity (Dopplergrams) on the surface of Sun almost uninterruptedly with high spatial resolution ( $0.5 \mathrm{arcsec} / \mathrm{pixel}$ ) and high cadence (45 sec) (Schou et al., 2010). The flare of February 15, 2011, was the first X-class flare of the new solar cycle, 24, and the first observed by HMI. This paper presents results of the initial analysis, which revealed the sunquake event. This event shows some curious properties, which make it different from the previously observed 'sunquakes'.

\section{Results}

The X2.2 flare of February 15, 2011, occurred in the central sunspot of active region NOAA 1158, which had a $\delta$-type magnetic configuration (Fig. 1). According to the GOES-15 soft X-ray measurements, the flare started at 01:44 UT, reached maximum at 01:56 UT and ended at 02:06 UT. The flare signals are clearly detected in all HMI observables, and show that the flare had a typical two-ribbon structure with the ribbons located on both sides of the magnetic neutral line. This is well seen in the magnetograms (Fig. 17). We consider two places of strong localized photospheric 
impacts as 'Impact 1' and 'Impact 2' for further analysis. The sunquake originated from 'Impact 1 '.

The sunquake event was initially revealed in the running difference movie of the raw Doppler velocity data (Kosovichev, 2011a). However, the wave structure is better seen after applying to the data a Gaussian frequency filter with a central frequency of $6 \mathrm{mHz}$ and a characteristic width of $2 \mathrm{mHz}$. This filter enhances the high-frequency sunquake signal relative to the lower-frequency background solar granulation noise. In addition, the images are remapped onto the heliographic Carrington coordinates using the Postel's azimuthal equidistant projection and tracked with the differential rotation rate. Figure 2 shows two frames of the frequency-filtered Doppler-velocity movie (available as supplementary online-only material). The sunquake wave appears about 20 minutes after the initial flare impact of the photosphere. The wave front has a circular shape, but it is not isotropic. The wavefront traveling outside the magnetic region in the North-East direction ('Wave 1') has the highest amplitude, and is most clearly visible. In the opposite direction the wave travels through a sunspot ('Wave 2'), and its amplitude is suppressed. Also, the wave front traveling through the sunspot is visibly delayed relative to 'Wave 1' traveling outside. Figure 3 shows positions of the two wave fronts at 02:08 UT in the corresponding magnetogram and whitelight images.

Figure 4 shows the time-distance diagrams obtained by remapping the frequency-filtered Dopplergrams onto the polar coordinates with the center at 'Impact 1', and averaging over the range of angles corresponding to the two parts of the wave fronts in Fig. 2, In these diagrams, the helioseismic acoustic waves form characteristic ridges, the slope of which corresponds to the local group-speed of the wave packets traveling between two surface points through the interior. The speed increases with the distance because for larger distances the waves travel through the deeper interior where the sound-speed is higher (e.g. Kosovichev, 2011b). For comparison, the theoretical travel times calculated in the ray approximation are shown by dashed curves. The starting points of these curves are chosen to approximately match the position of the ridges. Evidently, the ridge of 'Wave 2' is much weaker and shorter than the ridge of 'Wave 1'. In these diagrams, the wave source ('Impact 1') produces strong variations at zero distance at about 01:50 UT. Also, the 'Wave 2 ' front is delayed by $\sim 100 \mathrm{sec}$ with respect to 'Wave 1 '.

The time-distance diagrams show two interesting features. During approximately the first three minutes the wave source is moving in the direction of 'Wave 1' (Fig. 4) with a speed of about $15-17 \mathrm{~km} / \mathrm{s}$, which is higher than the local sound speed but may correspond to the magnetoacoustic speed of the sunspot penumbra in the vicinity of the source. The source motions, which can be supersonic, have been observed for other 'sunquake' events (Kosovichev, 2006b., , 2007). Similar to this case, the source motions are at least partly responsible for the anisotropy of the wave amplitude. In the previous cases the source motion was associated with apparent motions of the point-like photospheric impacts in the flare ribbons. In this case, the source motion may be associated with MHD waves excited by the flare momentum impact in the almost horizontal field on the penumbra. However, this process requires a special separate investigation. 
The sunquake source is associated with one of the impacts located along the flare ribbons. The flare ribbons consist of individual patches representing impacts of flare impulses. In these data it is easy to find that the location of the sunquake source was in the penumbra area near the edge of the active region. This area is identified as 'Impact 1' in Figures 13. It is characterized by strong and rapid variations of the Doppler velocity and magnetic field, and also by an impulsive increase of the continuum intensity (Fig. $5 \mathrm{~b}$ ). There were strong photospheric impacts in several other locations. However, these impacts did not provide clearly visible seismic waves. The reason for this is not clear. For comparison, in Figure $5 \mathrm{~b}$ we show the variations in one of the strongest compact impacts identified as 'Impact 2'. This impact was located in a region of strong magnetic field in the sunspot outer umbra near the magnetic neutral line. During the impact, the HMI data show a strong increase of the Doppler velocity, indicating downflows, a sharp impulsive decrease of the magnetic field strength, which relaxed to a value lower the the pre-flare strength, and an increase in the continuum intensity brightness. All the variations in 'Impact 2' are stronger than in 'Impact 1', but this impact did not generate strong sunquake ripples. It seems that the main difference between these two places of the flare impact onto the photosphere of the Sun is that 'Impact 1' was located in a region of relatively weak ( $\sim 400 \mathrm{G})$ magnetic field contrary to 'Impact 2 ', which was in strong field $(\sim 2000 \mathrm{G})$. In addition, 'Impact 1' was more variable and moving. It started near the inner boundary of the penumbra (bright point at the 'Impact 1' arrow in Fig. 3a) and then moved into the penumbra, generating a localized motion in this part of the penumbra. The dynamic nature of the flare impact seems to be important for understanding the mechanism of sunquakes. The strong magnetic field in 'Impact 2' probably restricted wave motions, and, perhaps, this may explain the absence of helioseismic response from this impact.

The origin of photospheric impacts during the flare impulsive phase is yet to be understood. In this case, it is particularly puzzling that the initial 'Impact 1' occurred in the early impulsive phase, prior to the hard X-ray impulse in the energy range of 50-100 keV, and just at the beginning of the X-ray 25-50 keV impulse (Fig. 5a). The traditional 'thick-target' mechanism of the energy transport in solar flares (e.g. Hudson, 2011) assumes that most of the energy is released in the form of high-energy electrons, which heat the solar chromosphere generating a localized highpressure zone. This zone explodes, and causes 'chromospheric evaporation' into the corona and the hydrodynamic impact in the photosphere, which leads to 'sunquake'. However, in this case the photospheric impact apparently happened before the main particle acceleration phase. This requires a new mechanism of the energy and momentum transport into the low atmosphere during the early 'pre-heating' flare phase. Certainly, further investigations of the sunquake events, their energetics and dynamics, will provide new insight in the mechanisms of the flare energy release and transport. 


\section{Discussion}

The first observations of the sunquake event from SDO/HMI revealed very interesting properties of the flare impact onto the solar photosphere. The HMI data with the significantly higher resolution than the previous $\mathrm{SOHO} / \mathrm{MDI}$ observations of sunquakes provide a new insight into the dynamics of the flare impact and the sunquake source. The preliminary analysis indicates that seismic flare waves are generated by the impact in the region of a relatively weak magnetic field of the sunspot penumbra. A significantly stronger impact in a region of high magnetic field strength did not generate helioseismic waves of a comparable magnitude.

A characteristic feature of this sunquake is anisotropy of the wave front: the observed wave amplitude is much stronger in one direction than in the others. This was observed also in previous events. In particular, the seismic waves excited during the October 28, 2003, flare had the greatest amplitude in the direction of the expanding flare ribbons. The wave anisotropy was attributed to the moving source of the hydrodynamic impact, which is located in the flare ribbons (Kosovichev, $2006 \mathrm{~b}, \mathrm{c}$ ). The motion of flare ribbons is often interpreted as a result of the magnetic reconnection processes in the corona. When the reconnection region moves up it involves higher magnetic loops, the footpoints of which are further apart. This may explain the expanding flare ribbons (as places of the photospheric flare impacts) and the association of sunquakes with the ribbon sources. In this event, the sunquake had a similar dynamical property: it started at an inner boundary of the sunspot penumbra and then quickly moved in the penumbra region. This was accompanied by a motion of this part of the penumbra. This is certainly an interesting phenomenon, which requires further investigation. Of course, there might be other reasons for the anisotropy of the wave front, such as inhomogeneities in temperature, magnetic field and plasma flows. However, the source motion seems to be quite important for generating sunquakes. In addition, the wave front traveling through the sunspot umbra is significantly delayed relative to the wave front traveling outside the sunspot. This delay may be related to the source motion and also to a lower wave speed in the sunspot umbra. Theoretical MHD modeling of the dynamic impact source and the wave propagation in sunspot models is necessary for the understanding of this phenomenon.

The comparison of the SDO/HMI observations with the X-ray observations from RHESSI shows that the photospheric impact, which led to the excitation of the helioseismic waves, occurred at the beginning of the flare impulsive phase, before the hard X-ray impulse in the energy range of 50-100 keV and before main particle acceleration phase. Perhaps, the energy transport into the lower atmosphere may be provided by the saturated heat flux as recently suggested for chromospheric evaporation by Battaglia et al. (2009). Theoretical models of the heat flux-saturated (or flux-limited) energy transport in the solar atmosphere were previously studied by several authors (e.g. Smith, 1986; Karpen \& Devore, 1987). Kosovichev (1988) showed that this transport has wave properties with a sharp shock-like heat front. Alternatively, the difference in timing between the photospheric impact and the hard X-ray flux may be related to changes in anisotropy of high-energy electrons during the flare impulsive phase (V. Petrosian, private communication; e.g.

Leach et al., 1985). It will be important to further investigate the role of high-energy particles, 
thermal and MHD effects in the initial phase of solar flares.

\section{REFERENCES}

Battaglia, M., Fletcher, L., \& Benz, A. O. 2009, A\&A, 498, 891

Ciaravella, A., Peres, G., \& Serio, S. 1991, Sol. Phys., 132, 279

Donea, A., Braun, D. C., \& Lindsey, C. 1999, ApJ, 513, L143

Donea, A. \& Lindsey, C. 2005, ApJ, 630, 1168

Hudson, H. S., Fisher, G. H., \& Welsch, B. T. 2008, in Astronomical Society of the Pacific Conference Series, Vol. 383, Subsurface and Atmospheric Influences on Solar Activity, ed. R. Howe, R. W. Komm, K. S. Balasubramaniam, \& G. J. D. Petrie , 221

Hudson, H. S. 2011, Space Sci. Rev., 7

Karpen, J. T., \& Devore, C. R. 1987, ApJ, 320, 904

Kosovichev, A. G. 1988, Soviet Astronomy Letters, 14, 243

Kosovichev, A. G. \& Zharkova, V. V. 1998, Nature, 393, 317

Kosovichev, A. G. 2006a, in Astronomical Society of the Pacific Conference Series, Vol. 354, Solar MHD Theory and Observations: A High Spatial Resolution Perspective, ed. J. Leibacher, R. F. Stein, \& H. Uitenbroek, 154

Kosovichev, A. G. 2006b, Sol. Phys., 238, 1

Kosovichev, A. G. 2006c, in ESA Special Publication, Vol. 624, Proceedings of SOHO 18/GONG 2006/HELAS I, Beyond the spherical Sun.

Kosovichev, A. G. 2007, ApJ, 670, L65

Kosovichev, A. G. 2011a, arXiv:1102.3954

Kosovichev, A. G. 2011b, Pulsation of the Sun and Stars, Lecture Notes in Physics, Vol. 832, Rozelot, Jean-Pierre; Neiner, Coralie (Eds.), 2011, (in press), arXiv:1103.1707

Kostiuk, N. D. \& Pikelner, S. B. 1975, Soviet Ast., 18, 590

Leach, J., Petrosian, V., \& Emslie, A. G. 1985, Sol. Phys., 96, 331

Schou, J., Borrero, J. M., Norton, A. A., Tomczyk, S., Elmore, D., \& Card, G. L. 2010, Sol. Phys., 196 
Smith, D. F. 1986, ApJ, 302, 836

Zharkova, V. V., \& Zharkov, S. I. 2007, ApJ, 664, 573

This preprint was prepared with the AAS IATEX macros v5.2. 


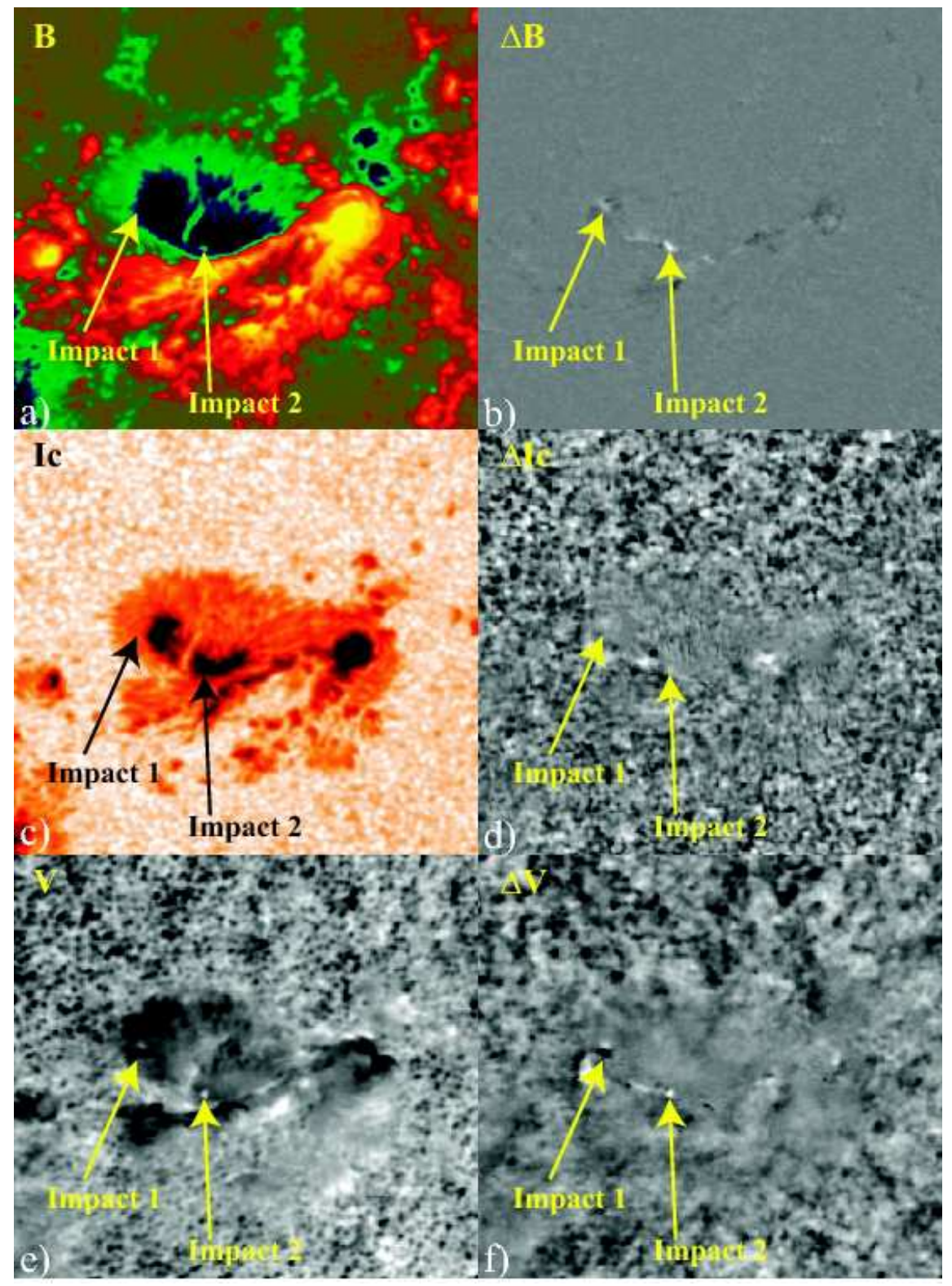

Fig. 1.- a) Images of AR 1158 during the impulsive phase of the X2.2 flare, at 2011.02.15 01:54:39 TAI: a) line-of-sight magnetic field $B$, c) continuum intensity $I_{c}$, and e) Doppler velocity $V$. The right panels show the differences between these images and the corresponding images taken $45 \mathrm{sec}$ earlier. The range of the magnetogram color map is $\pm 1 \mathrm{kG}$; the range of the Dopplergram is $\pm 1 \mathrm{~km} / \mathrm{s}$. Arrows show positions of two analyzed sources of transient flare variations located along the same flare ribbon. Traces of the second ribbon can be seen in panel b) to the right and below of 'Impact 2'. A powerful sunquake originated from 'Impact 1'. 'Impact 2' is a place of a strong impulsive impact, but it did not generate a significant sunquake. 


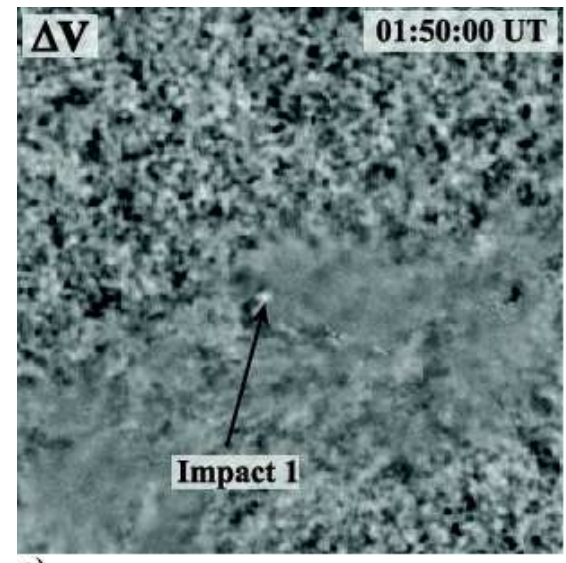

a)

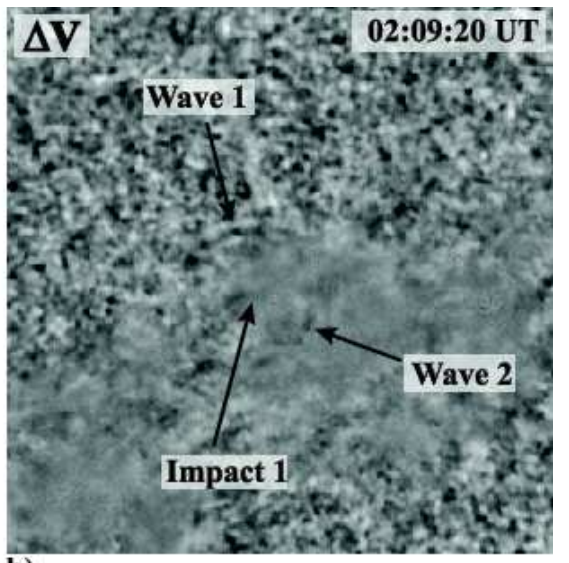

b)

Fig. 2.- Frequency-filtered Doppler velocity differences, $\Delta V$, at the moments of: a) the flare impact at 'Impact 1', calculated for 01:50:00 UT, and b) about 19 min later at 02:09:20 UT, showing the sunquake wavefront. These are two frames of the supplementary on-line movie. The movie is produced by interpolating the 45 -sec cadence data into a new series with 20 -sec cadence starting at 01:40 UT. This makes the movie slower and easier to watch. The original 45-sec cadence movie is available on-line in a RHESSI Science Nuggets article (Kosovichev, 2011a).

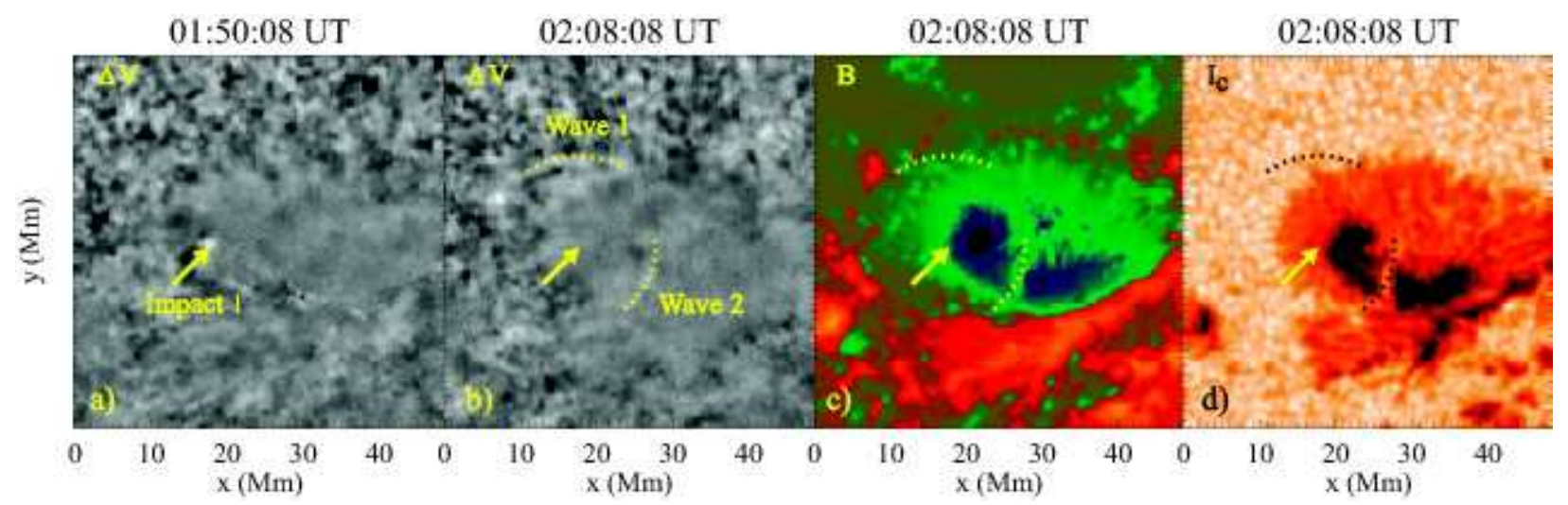

Fig. 3.- Illustration of positions of the flare 'Impact 1' (panel a) and the helioseismic fronts (b) observed in the Doppler-shift data, $\Delta V$, in the corresponding magnetogram, $B$, (c) and continuum intensity, $I_{c},(\mathrm{~d})$ images. 

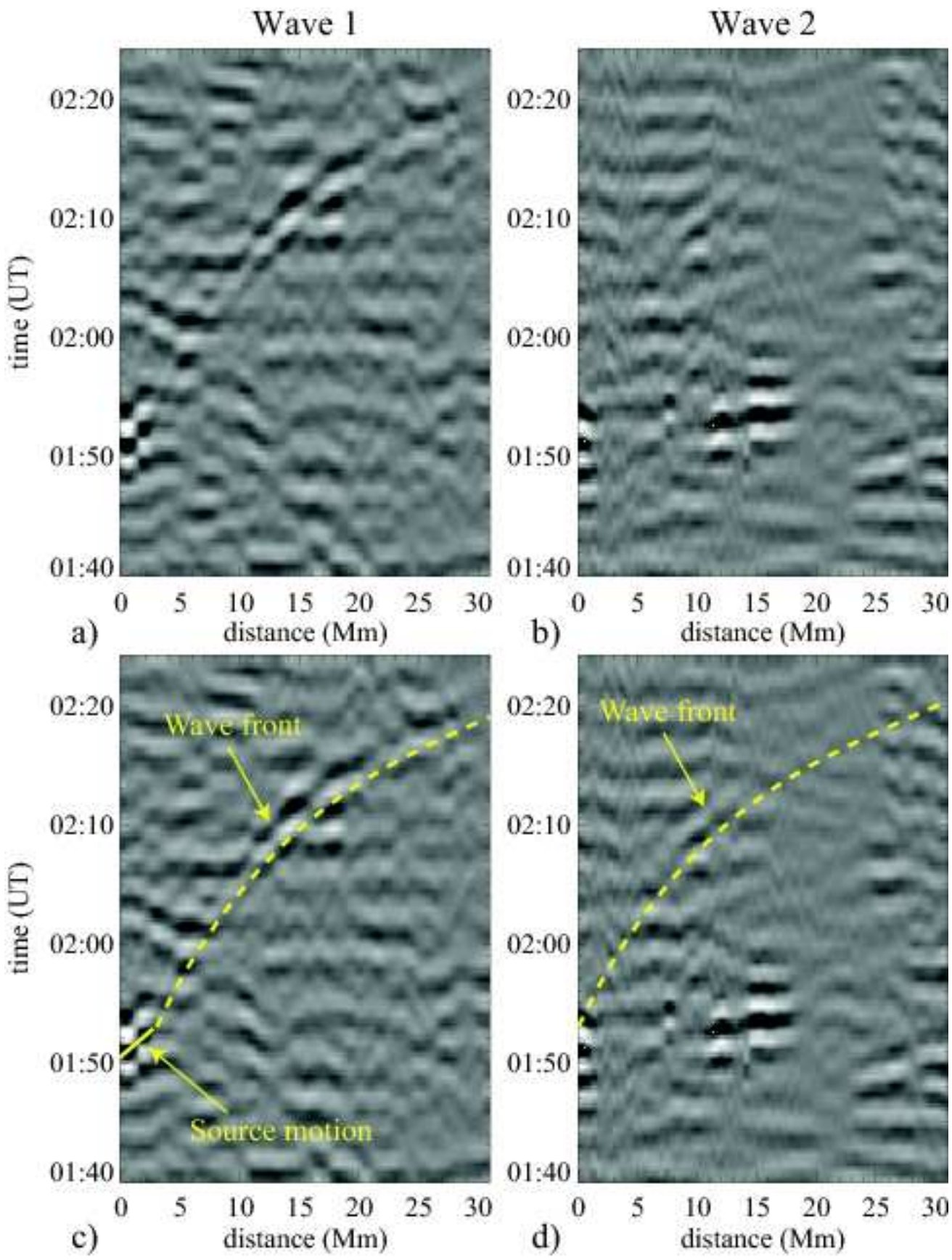

Fig. 4.- Time-distance diagrams for: a) 'Wave 1' and b) 'Wave 2', both originating from 'Impact 1'. In the duplicated diagrams c) and d), the dashed curves are the theoretical time-distance calculated for a standard solar model in the ray approximation. The locations of these curves are chosen to approximately match the leading wave fronts. The short solid line in panel c) indicates the source motion. 

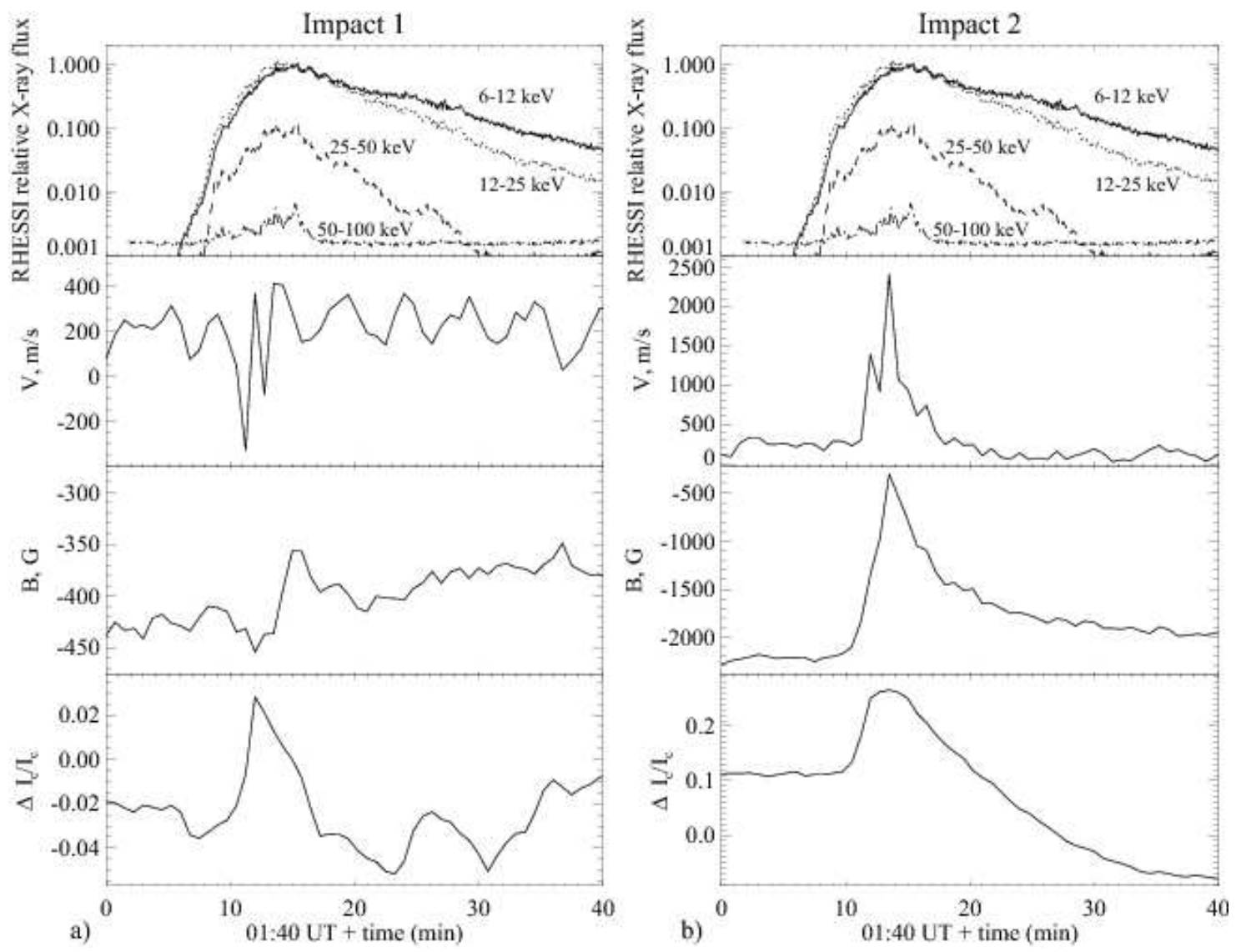

Fig. 5.- Variations of the total X-ray fluxes from RHESSI (relative to the 6-12 keV flux), Doppler velocity, magnetic field and continuum intensity at: a) 'Impact 1'; b) 'Impact 2'. The X-ray fluxes are integrated for the whole flare region, and thus are identical in panels a) and b). 